\title{
Study of cervical muscle response and injury of driver during a frontal vehicle collision
}

\author{
Zhenhai Gao ${ }^{\mathrm{a}, \mathrm{b}, \mathrm{c}}$, Chuzhao $\mathrm{Li}^{\mathrm{a}}$, Hongyu Hu${ }^{\mathrm{a},{ }^{*}}$, Hui Zhao ${ }^{\mathrm{b}, \mathrm{c}}$, Chaoyang Chen ${ }^{\mathrm{d}}$ and Huili Yu $\mathrm{u}^{\mathrm{b}, \mathrm{c}}$ \\ ${ }^{a}$ State Key Laboratory of Automobile Simulation and Control, Jilin University, Changchun 130025, \\ P.R. China \\ ${ }^{b}$ State Key Laboratory of Vehicle NVH and Safety Technology, Changan Automobile Holding Ltd, \\ Chongqing 401120, P.R. China \\ ${ }^{c}$ Changan Automobile Holding Ltd. Automotive Engineering Institute, Chongqing 401120, P.R. China \\ ${ }^{d}$ Spine Research Laboratory, Department of Biomedical Engineering, Wayne State University, Detroit, \\ MI 48201, USA
}

\begin{abstract}
Frontal vehicle collisions can cause injury to a driver's cervical muscles resulting from intense changes in muscle strain and muscle load. This study investigated the influence of collision forces in a sled test environment using a modified Hybrid III $50^{\text {th }}$ percentile dummy equipped with simulated spring-type muscles. Cervical muscle responses including strain and load of the sternocleidomastoid (SCM), splenius capitis (SPL), and trapezius (TRP) were analyzed, and muscle injury was assessed. The SCM, SPL, and TRP suffered average peak muscle strains of $21 \%, 40 \%$, and $23 \%$, respectively, exceeding the injury threshold. The average peak muscle loads of the SCM, SPL and TRP were $11 \mathrm{~N}, 25 \mathrm{~N}$, and $25 \mathrm{~N}$, respectively, lower than the ultimate failure load. The SPL endured the largest injury, while the injuries to the SCM and TRP were relatively small. This is a preliminary study to assess the cervical muscle of driver during a frontal vehicle collision. This study provides a foundation for investigating the muscle response and injury in sled test environments, which can lead to the improvement of occupant protections.
\end{abstract}

Keywords: Cervical muscle, muscle strain, muscle load, frontal collision, dummy

\section{Introduction}

Injuries to the head and neck resulting from collisions greatly impact a victim's daily life [1]. Out of all long-term chronic impairments from road accident injuries, 50\% occur in the neck [2]. Therefore, attention to biomechanical responses and injuries of the head and neck during collisions has increased.

Head and neck injuries commonly occur because of the interaction between the head and the airbag, as well as between the safety belt and the torso. Common types of collision-related neck injuries include compression-flexion, compression-extension, stretch-flexion, stretch-extension, and lateral bending. These injuries, which cause bone fractures, can also lead to injuries of soft tissues like ligaments and muscles [3]. Although muscle injuries are generally minor, they are the major cause of

\footnotetext{
* Address for correspondence: Hongyu $\mathrm{Hu}$, State Key Laboratory of Automobile Simulation and Control, Jilin University, Changchun 130025, P.R. China. Tel.: +86 (0431) 85095090; Fax: +86 (0431) 85682227; E-mail: huhongyu_jlu@126.com.
}

0959-2989/15/\$35.00 @ 2015 - IOS Press and the authors. 
chronic cervical diseases.

Muscle injuries have two main causes, sharp active contraction and excessive passive stretch [4]. Cervical motion during collision has the potential to cause both of these phenomena, meaning that collisions can easily result in injury to the cervical muscles. Muscle contractions consist of concentric, isometric and eccentric contraction. All types of contraction can cause muscle injuries, but eccentric contraction leads to the most severe ones [5]. During collisions, cervical muscles commonly suffer isometric or eccentric contraction injuries, while concentric contraction injuries rarely occur.

Researchers use techniques including cervical finite element modeling, cadaver cervical modeling, and volunteer sled tests to study biomechanical responses and injuries in the head and neck during collision. Toyota Motor Corporation developed the active human finite element model with three dimensional geometry muscles, which is able to study the active mechanical characteristics of muscles and enable simulation analysis [6]. Hedenstierna, et al. developed a cervical finite element model with human-like muscle distribution and mechanical characteristics to investigate muscle strain during collision [7]. Cervical finite element models with vertebrae, muscles, and ligaments can accurately describe physical responses and injuries in the head and neck. Ivancic, et al. used the neck from a cadaver to develop a dynamic whole cervical spine whiplash model that replicates anterior, lateral, and posterior muscle forces. This model's muscle force replication can produce dynamic responses that are nearly identical to those in the human body. Ivancic, et al. evaluated the performance of restraint systems during whiplash by combining their whole cervical spine model with a BioRID (Biomechanical Rear Impact Dummy) II dummy [8]. However, this model did not consider the active characteristics of muscles, and it was unable to measure changes in muscle length and muscle force. Because mechanical characteristics inside the human body cannot be fully described by these models, biomechanics research often involves volunteer sled tests. Cervical muscle strain during collision can only be indirectly acquired due to research ethics; therefore, a high speed motion capture system records muscle motions, allowing for the subsequent calculation of strain and assessment of muscle injuries [9].

Using a modified dummy with an applied muscle model, this study performed frontal sled tests at low velocity and measured the strain of cervical muscles during collision were measured. Then the muscle loads were calculated, considering the mechanical characteristics of spring-type muscles. Finally, the strain and load of muscles were used to assess the injury of cervical muscles.

\section{Methods}

\subsection{Selection of muscles}

The cervical muscles can be divided into two groups according to location: the anterior muscles, including platysma, sternocleidomastoid, and longus colli; and the posterior muscles, including splenius capitis, trapezius, and semispinalis. Many muscles are located around the neck, so researchers generally select several major ones to study. For example, one study looked at cervical kinematics during collision and demonstrated that the sternocleidomastoid, splenius capitis, and trapezius play important roles in cervical motion on the sagittal, coronal, and oblique planes [10]. This research also studies the sternocleidomastoid, splenius capitis, and trapezius.

The following abbreviations will be used in this paper: sternocleidomastoid, SCM; splenius capitis, SPL; and trapezius, TRP. 


\subsection{Modified dummy with applied muscle model}

During collisions, the head's momentum and kinetic energy will vary due to different weights, resulting in different muscle strains and muscle loads. However, this study did not consider the influence of head weight as an important factor. Thus, this study used the Hybrid III $50^{\text {th }}$ percentile dummy, which is the most widely-used dummy in automotive engineering. The dummy weighs 78.15 $\mathrm{kg}$ in total and $4.54 \mathrm{~kg}$ in the head, which are representative figures for the average body size of an adult male. Future studies will use Hybrid III $5^{\text {th }}$ and $95^{\text {th }}$ percentile dummies, which represent small and large human body sizes, to investigate the influence of head weight on muscle strain and muscle load.

A modified dummy with an applied muscle model was used to study muscle response characteristics and assess injuries. This dummy was created by equipping the Hybrid III $50^{\text {th }}$ percentile dummy with an applied muscle model consisting of the spring-type muscle module and muscle fixtures, described below.

The spring-type muscle module includes three pairs of extension springs which represent the SCM, SPL, and TRP, respectively. The term "Hill-type model" is commonly used to describe the mechanical characteristics of muscles. In this model, the combination of active and passive tension creates muscle force or tension [11]. The extension spring produces a reaction force while being stretched, similar to the mechanical characteristics produced by a muscle's passive component. Meanwhile, the spring can exert initial tension, representing the active tension produced by the active component of a muscle.

The extension springs in the spring-type muscle module are based on a simplification and fitting of the Hill-type model, considering the similarities of mechanical characteristics between the extension springs and the Hill-type model. These springs represent the combination of active and passive forces, but only the passive force changes during collision. Therefore, the active force stands constant, and the passive force increases with deflection, bringing a reaction force to the motion of the head and neck. The total tension of spring-type muscles consists of the constant active force and the variable passive force.

Muscle fixtures were used to connect the spring-type muscle module to the Hybrid III $50^{\text {th }}$ percentile dummy. The springs representing the SCM and SPL were connected through the upper neck ring and the lower neck ring, and the springs representing the TRP were connected between the lower neck ring and the shoulder ring. These muscle fixtures ensured the accuracy of the muscles' origin and insertion locations and avoided interactions between the spring-type muscle module and the neck structure of the dummy. The muscle fixtures only induced a slight influence on the collision response.

The currently available Hybrid III $50^{\text {th }}$ percentile dummy possesses the basic structure of a human body, including a skeleton and skin, and it has good biofidelity. However, significant differences remain between responses in the dummy and a human, such as the muscle activation state that occurs when a driver faces an emergency, which is not incorporated in the Hybrid III $50^{\text {th }}$ percentile dummy. A modified dummy was used to replicate accurately the muscle activation generated by a human's instinctual physiological reaction when facing imminent collision. This dummy included an independent muscle model that considers active and passive mechanical characteristics together. This applied muscle model designed to include the driver's instinctual physiological reaction was installed outside the cervical structure of the existing dummy, leaving the existing structure intact, compared to the model used by Hedenstierna, et al. and the whole cervical model designed by Ivancic other models $[7,8]$. 

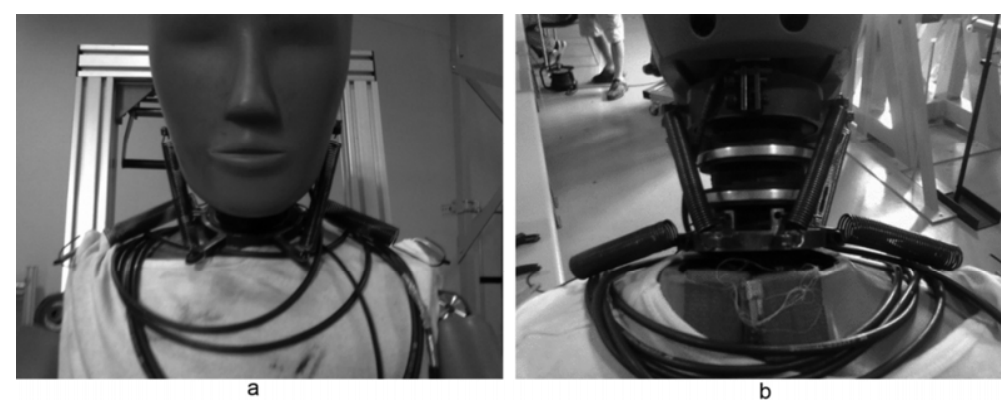

Fig. 1. The modified dummy with applied muscle model: (a) elevation view; (b) rear view.

This modified model also has certain limitations. First, differences of mechanical characteristics exist between the extension spring and the muscle, as the spring's parameters are derived from an approximation of the simplified Hill model; however, this is the most effective method to simulate the mechanical characteristics of muscles in sled test conditions. Second, the muscle model was designed based on muscle activation characteristics acquired from a series of tests simulating collisions under $25 \mathrm{~km} / \mathrm{h}$, suggesting that the muscle model should be designed differently for sled tests at other frontal collision speeds. Future studies will consider this design aspect and take the influence of collision speed into account.

Figure 1 shows the modified dummy with the applied muscle model.

\subsection{Sled tests}

The objects of this study were to analyze muscle response characteristics and to assess injuries, so six sled tests were performed using the modified dummy with the applied muscle model. During the tests, the dummy was positioned in a seat with a safety belt. The characteristics of the seat and belt were controlled to account for their influence over the collision response; the tests used a rigid seat and a general safety belt, which eliminated the influence of a seat cushion, belt force limitation, and belt pre-tension. The sled tests used lower velocities because the load cells integrated within the dummy are easily damaged. In summary, the sled tests were controlled as follows: (1) $25 \mathrm{~km} / \mathrm{h}$ frontal collision; (2) general safety belt, with no force limiter or pre-tensioner; (3) rigid seat, with no headrest or cushion; and (4) modified dummy with muscle fixtures and spring-type muscle modules.

In this study, characteristics of cervical muscle response included muscle strain and muscle load. Muscle strain is defined as the variation between instant muscle length and static muscle length. Static muscle length, $\mathrm{L}_{0}$, is defined as the free length of the extension spring, while the instant muscle length, L', is defined as the instant distance between the origin and the insertion of the spring-type muscle. When the spring-type muscle is at the static muscle length, the initial tension of the spring is equal to the active muscle force, indicating that passive muscle force is not present if the spring is not stretched. Thus, the authors hypothesized that the static muscle length is equal to the muscle fiber length, $\mathrm{L}_{\mathrm{f}}$. Muscle deflection, $\Delta \mathrm{L}$, is defined as the difference between the instant muscle length and the static muscle length, calculated as $\Delta \mathrm{L}=\mathrm{L}^{\prime}-\mathrm{L}_{0}$. Therefore, the muscle deflection is positive if the spring-type muscle lengthens. Muscle strain is calculated as the ratio of the muscle deflection to the static muscle length. Muscle load, $\mathrm{F}$, is defined as the load that the spring-type muscle experiences during collision, or $\mathrm{F}=\mathrm{F}_{0}+\mathrm{k}^{*} \Delta \mathrm{L}$, where $\mathrm{F}_{0}$ is the active muscle force, $\mathrm{k}$ is the spring rate, and $\Delta \mathrm{L}$ is the deflection of the spring. $\mathrm{F}_{0}$ and $\mathrm{k}$ of SCM, SPL and TRP represent differences in the physiologic characteristics of muscles. 
Four high speed cameras recorded motions at the origin and insertion points of the cervical muscles during collision. The length of the spring-type muscles was conceptualized as the distance between the origin and the insertion points, so muscle strain could be calculated. The cameras were located in the left, right, left front, and right front of the collision area. The left-sided and right-sided muscle responses were averaged because they were nearly identical [9]. The cameras recorded motions within a span of $400 \mathrm{~ms}$. This period was divided into two phases for the purpose of analyzing cervical muscle response characteristics: (1) In phase 1 (P1), which ranged from $0 \mathrm{~ms}$ to 211 (24) ms, the dummy moved forward to the extreme position and then retracted to the initial position; (2) in phase 2 (P2), which ranged from 211 (24) ms to $400 \mathrm{~ms}$, the dummy moved backward to the extreme position and then rebounded to the initial position. Values are shown as mean (SD).

\subsection{Statistical analysis}

One-way analyses of variance (ANOVA) were performed to determine whether peak muscle strains and muscle loads differed significantly among muscles. If ANOVA found significant differences, post hoc pairwise comparisons were performed using the Turkey Honest Significant Difference method. In all analyses, the level of significance was set as 0.05 .

\section{Results}

\subsection{Muscle strain}

As Figure 2 shows, SCM strain decreased in P1 and then increased to its peak value in P2. Peak SCM strains ranged from $20 \%$ to $25 \%$. Unlike the SCM, SPL and TRP strains increased to their peak values in P1 and then began to decrease (Figure 2). The peak strains of SPL and TRP ranged from $36 \%$ to $47 \%$ and from $20 \%$ to $25 \%$, respectively. Strain values for SCM and SPL were negative in P1 and P2, respectively, meaning that the lengths of SCM and SPL were smaller than the static muscle lengths. The peak strain of the SPL was significantly larger than that of the SCM or TRP, but the differences between the SCM and TRP were not significant (see Table 1). During collision, the SCM, SPL and TRP strains ranged from $-18 \%$ to $25 \%,-6 \%$ to $47 \%$, and $12 \%$ to $25 \%$, respectively.

As Figure 2 shows, the SCM, SPL, and TRP began at a stretch of $7 \%(0 \%), 18 \%(1 \%)$, and $15 \%$ $(0 \%)$, respectively. The SCM was stretched from a relatively small strain in P1 to larger strain in P2, with the strain increasing from $-16 \%(2 \%)$ to $21 \%(3 \%)$ with a total variation of $36 \%(6 \%)$. In contrast, the SPL and TRP were both stretched to a large strain in P1. The strain on the SPL increased from $18 \%(1 \%)$ to $40 \%$ (3\%), with a variation of $23 \%(4 \%)$. The strain on the TRP increased from $15 \%$ $(0 \%)$ to $23 \%(2 \%)$, with a variation of $8 \%(2 \%)$. Values are shown as mean (SD).

The times of peak strains in the SPL and TRP occurred near the same point in P1, while the time of peak strain in the SCM occurred in P2. When the SPL and TRP were at their peak strains, the SCM was at its minimal strain. Conversely, the SCM was in its peak strain when the strains of the SPL and TRP were relatively small. This suggests that the motion phase of the SCM is opposite to those of the SPL and TRP.

\subsection{Muscle load}



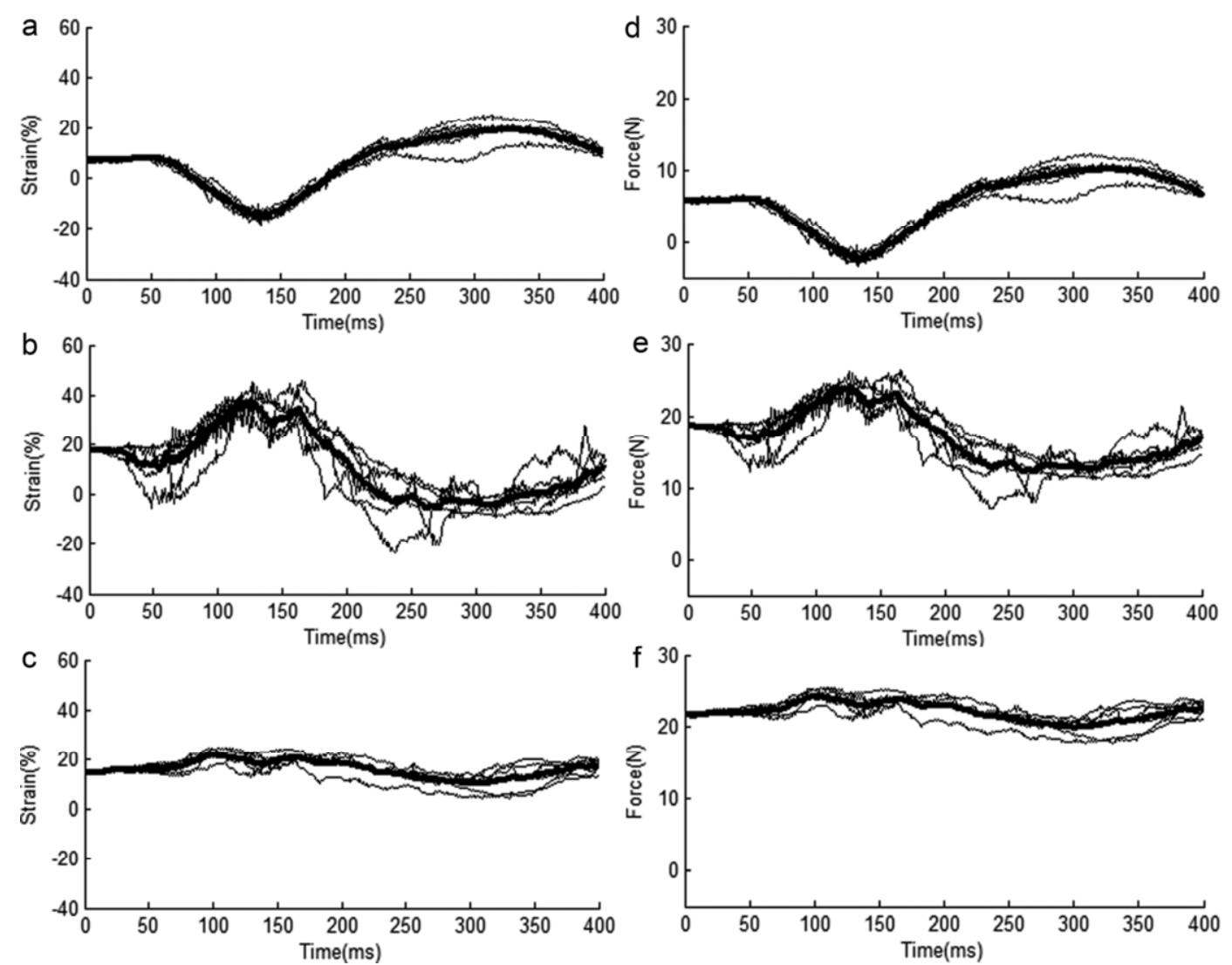

Fig. 2. Muscle strain and muscle load: a, b, and c represent the strain on the SCM, SPL, and TRP, respectively; e, f, and $g$ represent the load on the SCM, SPL, and TRP, respectively. Individual results are shown in thin lines, and the average value is shown as a thick line.

Table 1

Times of peak muscle strains and muscle loads

\begin{tabular}{llllll}
\hline & Strain & & & Force & \\
\cline { 2 - 3 } \cline { 5 - 6 } & Peak $(\%)$ & Time $(\mathrm{ms})$ & & Peak $(\mathrm{N})$ & Time $(\mathrm{ms})$ \\
\hline SCM & $20.53(3.41)$ & $317(17)$ & & $10.59(1.24) \#$ & $317(17)$ \\
SPL & $40.41(3.42)^{*}$ & $136(21)$ & & $24.85(0.95)$ & $136(21)$ \\
TRP & $22.54(1.86)$ & $123(30)$ & & $24.70(0.70)$ & $123(30)$
\end{tabular}

Note: Values are shown as mean (SD); *: SPL was significantly different from SCM and TRP; \#: SCM was significantly different from SPL and TRP.

The muscle load was calculated based on the muscle strain, so the motion phase and the time of response were identical to those discussed previously. The load on the SCM decreased in P1 and then increased to its peak value in P2, while the loads on the SPL and TRP increased to their peak values in P1 and then began to decrease (see Figure 2). Over the six tests, the peak loads of the SCM, SPL, and TRP ranged from $8 \mathrm{~N}$ to $13 \mathrm{~N}, 23 \mathrm{~N}$ to $27 \mathrm{~N}$, and $23 \mathrm{~N}$ to $26 \mathrm{~N}$, respectively. The peak load on the SCM was significantly smaller than those on the SPL and TRP, but the difference between the peak loaks of the SPL and TRP was not significant (see Table 1). During collision, the loads on the SCM, 
SPL, and TRP ranged from $-3 \mathrm{~N}$ to $13 \mathrm{~N}, 12 \mathrm{~N}$ to $27 \mathrm{~N}$, and $21 \mathrm{~N}$ to $26 \mathrm{~N}$, respectively.

The loads on the SCM, SPL, and TRP when the collision began were $6(0) \mathrm{N}, 19(0) \mathrm{N}$, and 22(0) N, respectively. The load on the SCM increased from a small strain in P1 to large strain in P2. During this process, the load increased from $-3(1) \mathrm{N}$ to 11(1) N. The loads on the SPL and TRP reached their greatest values in $\mathrm{P} 1$, increasing from $19(0) \mathrm{N}$ to $25(1) \mathrm{N}$ and from $22(0) \mathrm{N}$ to $25(1) \mathrm{N}$, respectively.

\section{Discussion}

\subsection{Muscle strain}

Research shows that eccentric muscle contractions result in force decreases and morphological evidence of injuries [12-15]. The force deficits are related to muscle strain [13-15] and muscle force $[12,14,15]$. Force deficits are also influenced by the initial muscle fiber length during the stretch [15].

In single lengthening contractions, strain as low as $5 \%$ to $10 \%$ can injure Type II muscle fibers, and higher strains may injure Type I fibers [16]. Studies of intact muscles usually apply strains of $20 \%$ to $25 \%$ to cause injury $[12,13]$. Strains of $30 \%$ to $50 \%$ induce significant force deficits [14]. Brooks [17] demonstrated that a single stretch of only $30 \%$ strain can produce a significant force deficit for active muscles, whereas a larger strain is required for passive muscles.

In the six sled tests, the strains on the SCM, SPL, and TRP were $20 \%$ to $25 \%, 36 \%$ to $47 \%$, and $20 \%$ to $25 \%$, respectively; all of these exceeded the threshold for muscle injuries, especially the average peak strain of the SPL, which reached $40 \%$ and caused a force deficit of at least $10 \%[14,17]$. The average peak strains of the SCM and TRP were lower, so the magnitudes of the force deficits were smaller.

It remains unknown whether more severe injuries result from longer or shorter initial fiber lengths. However, the magnitude of a force deficit increases with an increase in relative displacement [15]. At the beginning of collision, a muscle suffers an active force and a certain passive force. These induce initial strains and initial loads that are different for each muscle. The cervical flexion in P1 caused the SCM to stretch to its peak strain from a lower strain value than it began with. The SPL and TRP were stretched from their initial lengths, so the variations in strains were small. These results suggest that the SCM might suffer more severe injuries than the SPL or TRP.

The SCM strains were larger in P2, while the strains of the SPL and TRP were larger in P1, meaning that the SCM showed a reverse motion phase to that of the SPL and TRP. This is a result of the physiological structure of the muscles. The SCM is an anterior muscle and resists the cervical extension in P2, whereas the SPL and TRP are posterior muscles and resist the cervical flexion in P1.

The strains of the SCM and SPL were negative in P1 and P2, with intervals ranging from 86 (7) ms to 184 (3) ms and from 235 (32) ms to 338 (43) ms, respectively. These indicate that the muscle lengths during these intervals were smaller than the static muscle lengths, but not that the muscles were shortening. Rather, the likely cause is the limitation of the extension spring itself, which does not generate force or even deform when the instant length is smaller than the free length. A small subsidence existed after the peak strain of the SPL, but this cannot be well explained. Further studies must investigate and eliminate these limitations.

\subsection{Muscle load}

Muscles are injured when the muscle load is greater than the ultimate failure load. Muscle injury 
can even occur under $70 \%$ of the ultimate failure load [18]. Because stretch tests on the human body are restricted for ethical reasons, the ultimate failure load is calculated from animal testing. Mathematical models can also estimate the maximal isometric force for different muscles [19]. The inputs of these models are the maximal force of the neck in different directions, which can be measured in volunteers.

Separate from the estimations achieved with volunteers and mathematical models, cervical muscle loads during collision can be directly measured using the modified dummy from this study. The average peak muscle loads for the SCM, SPL, and TRP are 11(1) N, 25(1) N, and 25(1) N, respectively (see Table 1). These values are lower than the ultimate failure loads, which are amplified according to differences in physiological characteristics $[18,20]$. These are even lower than the maximal isometric forces estimated by the biomechanical model, $170 \mathrm{~N}$ for the SCM, $68 \mathrm{~N}$ for the SPL, and $81 \mathrm{~N}$ for the TRP [19]. These results indicate that the SCM and SPL can suffer greater strains and muscle loads during collision.

\section{Conclusions}

During collision, cervical muscles are exerted to protect the driver's head and neck from injury. Cervical muscles can be injured at the same time. This study performed low-velocity frontal sled tests using a modified dummy with an applied muscle model to measure muscle strains. Subsequently, muscle loads were calculated based on the mechanical characteristics of spring-type muscles. Finally, cervical muscle injuries were assessed using the muscle strain and muscle load data. This is a preliminary study to assess the cervical muscle of driver during a frontal vehicle collision, and more works will be performed in the future. This study found that:

(1) The average peak muscle strains of the SCM, SPL, and TRP were $21 \%, 40 \%$, and $23 \%$, exceeding the threshold for injury.

(2) The average peak muscle loads of the SCM, SPL, and TRP were $11 \mathrm{~N}, 25 \mathrm{~N}$, and $25 \mathrm{~N}$, lower than the ultimate failure load.

(3) Simulated cervical muscles were injured during collision; the SPL suffered the greatest injury, while injuries to the SCM and TRP were relatively small.

\section{Acknowledgments}

This study was supported by the 973 Program under Grant No. 2012CB723802; Specialized Research Fund for the Doctoral Program of Higher Education under Grant No. 20120061110028; Jilin Provincial Research Foundation for Technology Guidance under Grant No. 20130413058GH and Grant No. 20150204055GX.

\section{References}

[1] J. Yang, Overview of research on injury biomechanics in car-pedestrian collisions, Chinese Journal of Automotive Engineering 1 (2011), 81-93.

[2] M. Von Koch, A. Nygren and C. Tingvall, Impairment pattern in passenger car crashes, a follow-up of injuries resulting in long-term consequences, Proceedings of the Fourteenth International Technical Conference on Enhanced Safety of Vehicles, Munich, Germany, 1994. 
[3] X. Li and L. Dai, Advances in biomechanical study on cervical spine injuries, Chinese Journal of Orthopaedic Trauma 6 (2004), 920-923

[4] G.Y. El-Khoury, E.A. Brandser, M.H. Kathol, D.S. Tearse and J.J. Callaghan, Imaging of muscle injuries, Skeletal Radiology 25 (1996), 3-11.

[5] A.J. Sargeant and P. Dolan, Human muscle function following prolonged eccentric exercise, European Journal of Applied Physiology and Occupational Physiology 56 (1987), 704-711.

[6] M. Iwamoto, Y. Nakahira and T. Sugiyama, Investigation of pre-impact bracing effects for injury outcome using an active human fe model with $3 \mathrm{~d}$ geometry of muscles, 22nd International Technical Conference on the Enhanced Safety Vehicles, Stuttgart, Germany, 2011, pp. 11-0150.

[7] S. Hedenstierna, P. Halldin, K. Brolin and H. von Holst, Development and evaluation of a continuum neck muscle model, Journal of Biomechanics 39 (2006), S150.

[8] P.C. Ivancic, M.M. Panjabi, S. Ito, P.A. Cripton and J.L. Wang, Biofidelic whole cervical spine model with muscle force replication for whiplash simulation, European Spine Journal 14 (2005), 346-355.

[9] J.R. Brault, G.P. Siegmund and J.B. Wheeler, Cervical muscle response during whiplash: Evidence of a lengthening muscle contraction, Clinical Biomechanics 15 (2000), 426-435.

[10] S. Kumar, Y. Narayan and T. Amell, Analysis of low velocity frontal impacts, Clinical Biomechanics 18 (2003), 694703.

[11] M. Nordin and V.H. Frankel, Biomechanics of skeletal muscle, in: Basic Biomechanics of the Musculoskeletal System, D. Leger, ed., Lippincott Williams \& Wilkins, Philadelphia, 2001, pp. 148-174.

[12] K.K. McCully and J.A. Faulkner, Characteristics of lengthening contractions associated with injury to skeletal muscle fibers, Journal of Applied Physiology 61 (1986), 293-299.

[13] R.L. Lieber and J. Friden, Muscle damage is not a function of muscle force but active muscle strain, Journal of Applied Physiology 74 (1993), 520-526.

[14] S.V. Brooks and J.A. Faulkner, Severity of contraction-induced injury is affected by velocity only during stretches of large strain, Journal of Applied Physiology 91 (2001), 661-666.

[15] K.D. Hunter and J.A. Faulkner, Pliometric contraction-induced injury of mouse skeletal muscle: Effect of initial length, Journal of Applied Physiology 82 (1997), 278-283.

[16] P.C. Macpherson, M.A, Schork and J.A. Faulkner. Contraction-induced injury to single fiber segments from fast and slow muscles of rats by single stretches, American Journal of Physiology-Cell Physiology 271 (1996), 1438-1446.

[17] S.V. Brooks, E. Zerba and J.A. Faulkner, Injury to muscle fibres after single stretches of passive and maximally stimulated muscles in mice, The Journal of Physiology 488 (1995), 459-469.

[18] C.T. Hasselman, T.M. Best, A.V. Seaber and W.E. Garrett, A threshold and continuum of injury during active stretch of rabbit skeletal muscle, The American Journal of Sports Medicine 23 (1995), 65-73.

[19] S.P. Moroney, A.B. Schultz and J.A. Miller, Analysis and measurement of neck loads, Journal of Orthopaedic Research 6 (1988), 713-720.

[20] L.K. Kamibayashi and F.J. Richmond, Morphometry of human neck muscles, Spine 23 (1998), 1314-1323. 